\title{
Esplenectomía laparoscópica mano asistida por esplenomegalia masiva
}

\author{
Hand-assisted laparoscopic splenectomy for massive \\ esplenomegaly - volume spleen \\ Yisvanth Pérez Ponce, ${ }^{*}$ Carlos Alberto Santana Pérez, ${ }^{\ddagger}$ \\ Luis Ángel Muciño Pérez, ${ }^{\ddagger}$ José Luis Gutiérrez Velazco ${ }^{\ddagger}$ \\ * FACS, Cirugía y Medicina de Alta Especialidad Sepsis, Obesidad y Cirugía Compleja AC. Cirugía General UAEMex. \\ ‡ Cirugía General.
}

Centro Médico ISSEMyM Toluca.

\section{RESUMEN}

Introducción: La cirugía de mínimo acceso ha evolucionado constantemente desde la década de 1980; múltiples centros a nivel internacional han desarrollado dispositivos y abordajes con la finalidad de minimizar el riesgo de sangrado, del dolor posoperatorio, de la estancia hospitalaria y los resultados estéticos. Dichos avances han catalogado al abordaje laparoscópico como la opción principal en la resección esplénica no urgente. Material y métodos: Se presenta el caso de paciente femenino de 74 años con múltiples comorbilidades, anemia crónica y dolor abdominal difuso, con predominio en cuadrante superior izquierdo, con esplenomegalia palpable hasta la cresta iliaca izquierda; ultrasonido y tomografía de abdomen con esplenomegalia $20 \times 17 \times 8 \mathrm{~cm}$ y volumen calculado de $1400 \mathrm{~cm}^{3}$. Esplenectomía mediante abordaje laparoscópico mano asistido con tres puertos y control vascular con endograpadora; bazo de $25 \times 15 \times 10 \mathrm{~cm}$. Resultados: Evolución satisfactoria con egreso al cuarto día de posoperatorio, con resolución del motivo de consulta. Conclusión: La valoración integral de los pacientes con esplenomegalia masiva facilita el tratamiento operatorio. La esplenectomía laparoscópica mano asistida (HALS) es una opción viable, permite adecuada

\section{ABSTRACT}

Introduction: Minimally invasive surgery has constantly evolved since the 1980's; multiple international centres have developed devices and approaches with the intention of reducing the risk of haemorrhage, postoperative pain and hospital stay, as well as improving cosmetic results. These improvements have positioned laparoscopic approach as the main option for the non - urgent splenic resection. Methods and material: The case presents a 74-year-old female with multiple comorbidities, chronic anemia and difuse abdominal pain, predominant in the upper left cuadrant; palpable splenomegaly down to the left iliac crest; US and CT shows a $20 \times 17 \times 8 \mathrm{~cm}$ spleen, with a calculated volume of $1400 \mathrm{~cm}^{3}$. Hand-assisted laparoscopic approach is performed through 3 ports; vascular control with endostapler, finding a $25 \times 15 \times 10 \mathrm{~cm}$ spleen. Results: Satisfactory postoperative evolution and patient discharge at day 4 with significant haemoglobin rise and cese of abdominal pain. Conclusion: Integral selection and meeting of criteria in patients with massive splenomegalia, improves the surgical treatment. Hand-assisted laparoscopic splenectomy (HALS) is a viable option, which allows an optimized movility of the spleen and adjacent structures and which

\section{www medigraphic org. \\ Recibido: 11/08/2020. Aceptado: 02/02/2021. \\ Correspondencia: Yisvanth Pérez Ponce FACS. \\ Avenida Vicente Guerrero Núm. 209, Torre II, Consultorio 306, \\ Col. Centro, 50120. Toluca Estado de México. Tel. (72) 2215-0550. \\ E-mail: dryisvanthperezponce@hotmail.com}

Citar como: Pérez PY, Santana PCA, Muciño PLÁ, Gutiérrez VJL. Esplenectomía laparoscópica mano asistida por esplenomegalia masiva. Rev Mex Cir Endoscop. 2020; 21 (3): 166-173. https://dx.doi.org/10.35366/99843 
movilización del bazo y estructuras adyacentes, disminuye el índice de conversión quirúrgica a cirugía abierta, menor morbilidad y conserva los beneficios de la cirugía de mínimo acceso.

Palabras clave: Esplenomegalia masiva, laparoscopía mano asistida, anemia, mínima invasión, púrpura trombocitopénica idiopática, esplenectomía. reduce the risk of surgical convertion to open surgery, less associated morbility and still preserves the benefits of minimally invasive surgery.

Keywords: Massive splenomegaly, hand-assisted laparoscopy, anemia, minimally invasive, idiopatic thrombocytopenic purpura, splenectomy.

\section{INTRODUCCIÓN}

La esplenectomía laparoscópica (EL) ha sido aceptada, al igual que la apendicectomía y la colecistectomía, como abordaje seguro y eficaz por minimizar el riesgo de sangrado; no requiere un tiempo reconstructivo, establece pasos críticos y referencias anatómicas precisas, además de ofrecer los beneficios del abordaje de mínimo acceso: menor dolor, menor tiempo de recuperación y beneficios estéticos; lo que coloca a este abordaje como la opción principal en la resección esplénica no urgente, descrita inicialmente por Delaitre B desde 1991.1,2

La cirugía laparoscópica mano asistida (HALS, hand assisted laparoscopic surgery, por sus siglas en inglés) es una opción actualmente aceptada porque conserva las ventajas de la cirugía de mínimo acceso, evita la pérdida de la sensación de profundidad y aumenta la sensación táctil. Es una variante del abordaje laparoscópico, en el que de forma planeada se efectúa una minilaparotomía que permite introducir una de las manos del cirujano o del ayudante en el interior del abdomen, mientras se mantiene el neumoperitoneo y se efectúan las maniobras de disección bajo control videoendoscópico, beneficiándose de la magnificación favorecida por la óptica. ${ }^{1-3}$

Se presenta caso de esplenectomía laparoscópica mano asistida en paciente con esplenomegalia masiva y se revisa la literatura asociada.

\section{PRESENTACIÓN DEL CASO}

Femenino de 74 años de edad con diabetes mellitus tipo 2 de cinco años de diagnóstico, enfermedad pulmonar obstructiva crónica (EPOC) con dependencia de oxígeno, cuadro depresivo, hipertensión arterial sistémica; histerectomía en 1984 por sangrado uterino anormal, tabaquismo dos cajetillas por día por 25 años.

Paciente en protocolo de estudio por anemia crónica, recibió valoración quirúrgica, hallazgo principal de sintomatología de sensación de pesantez en hemiabdomen izquierdo, con dolor abdominal difuso de predominio en cuadrante superior izquierdo, negando síntomas B. Refiere disnea ocasional a medianos esfuerzos en tratamiento médico y con apoyo nocturno de oxígeno suplementario. Sin referir dolor precordial, palpitaciones secundarias.

A la exploración física se encuentra consciente, orientada, cuello sin ingurgitación yugular, tórax simétrico, murmullo vesicular, a la auscultación se encuentra con discreta disminución de los ruidos respiratorios sin agregados, ruidos cardiacos rítmicos de adecuada intensidad y frecuencia, abdomen blando depresible, dolor a la palpación en flanco izquierdo y mesogastrio, asociado a esplenomegalia con matidez $10 \mathrm{~cm}$ por debajo de reborde costal izquierdo que se extiende a línea media, normoperistalsis, extremidades íntegras, sin edema, llenado capilar normal.

Laboratorios: Leucocitos: $2010^{3} / \mu \mathrm{L}$; neutrófilos: 93\%; hemoglobina: $9.9 \mathrm{~g} / \mathrm{dL}$; hematocrito: $30 \%$; plaquetas: 142 103/ $\mu \mathrm{L}$; glucosa: $188 \mathrm{mg} / \mathrm{dL}$; urea: $18 \mathrm{mg} / \mathrm{dL}$; BUN: $9 \mathrm{mg} /$ $\mathrm{dL}$; creatinina: $0.6 \mathrm{mg} / \mathrm{dL}$; sodio: $140 \mathrm{mmol} / \mathrm{L}$; potasio: $3.8 \mathrm{mmol} / \mathrm{L}$; ALT: $16 \mathrm{U} / \mathrm{L}$; albúmina: $4.1 \mathrm{~g} / \mathrm{dL}$; bilirrubina total: $1.4 \mathrm{mg} / \mathrm{dL}$; bilirrubina directa: $0.9 \mathrm{mg} / \mathrm{dL}$; fosfatasa alcalina: $107 \mathrm{U} / \mathrm{L}$.

De los estudios de imagen se realizó ultrasonido y tomografía axial computarizada abdominal, donde se observa esplenomegalia $20 \times 17 \times 8 \mathrm{~cm}$, con un volumen calculado de 1,400 $\mathrm{cm}^{3}$ (Figura 1). Se completó protocolo preoperatorio y se refirió como ASA: III, LEE: I, GOLDMAN I, NYHA: II, escala de Caprini: 5 puntos, DETSKY: I bajo riesgo.

\section{Tratamiento}

Ante el contexto de esplenomegalia masiva sin enfermedad hematológica subyacente aparente pero sintomática, con dolor abdominal persistente y datos de hiperesplenismo por anemia crónica y trombocitopenia discreta, se planea manejo quirúrgico con base en esplenectomía laparoscópica mano asistida. Bajo anestesia general, se colocó en posición decúbito supino con lateralización derecha, se realiza incisión paramedial supraumbilical izquierda de 8 $\mathrm{cm}$, se coloca retractor-protector de herida (Alexis) grande y puerto de mano (hand port) y se insufla neumoperitoneo hasta los $12 \mathrm{mmHg}$, se realiza laparoscopía identificando esplenomegalia de $35 \times 25 \times 6 \mathrm{~cm}$ (Figura 2).

Se colocaron tres puertos, uno de $10 \mathrm{~mm}$ en línea media clavicular, otro de $10 \mathrm{~mm}$ en línea axilar anterior 

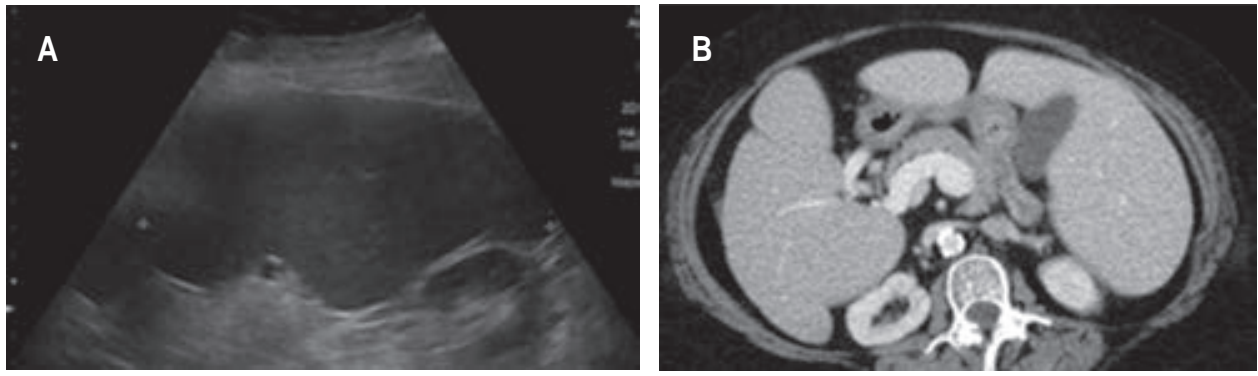

Figura 1:

A) Corte transversal ultrasonográfico que descarta patología infiltrativa.

B) Corte axial de tomografía contrastada con bazo de gran volumen que se aproxima a la línea media. C) Corte coronal con bazo de gran tamaño y su relación

con la cresta iliaca. D) Corte sagital que muestra la ocupación anteroposterior del bazo.
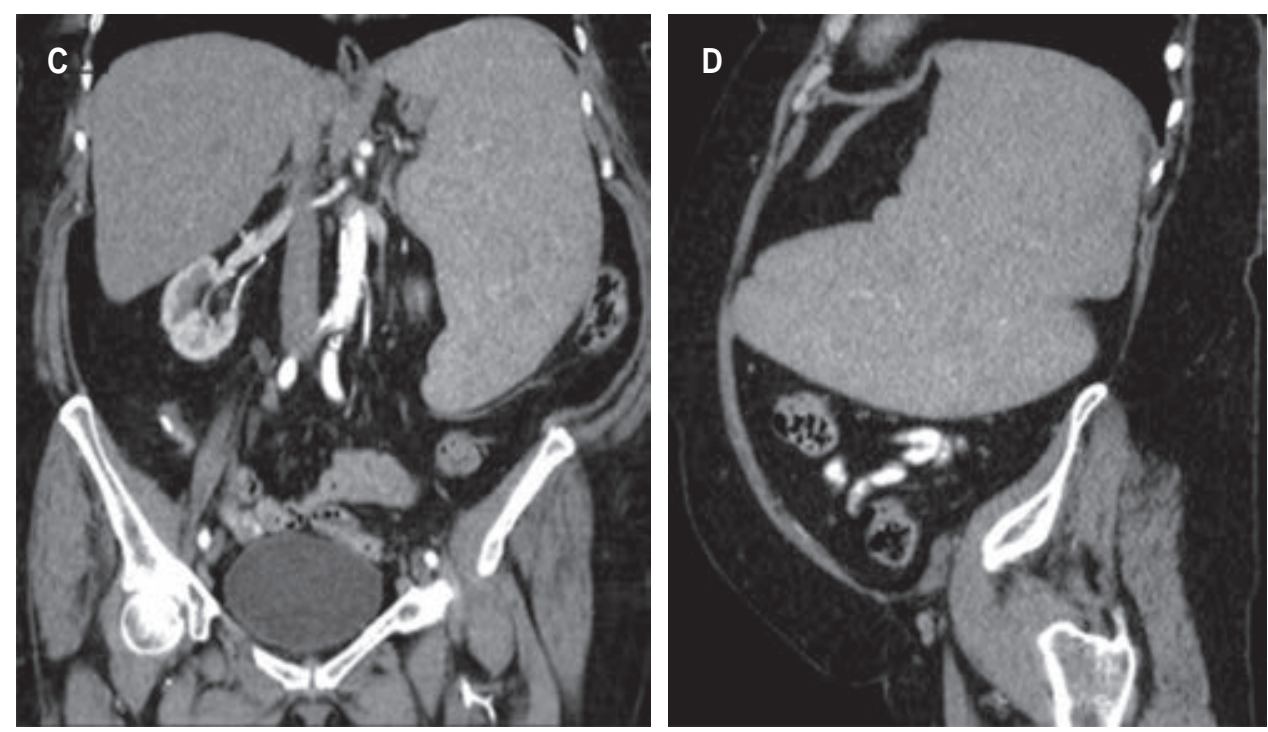

y uno de $5 \mathrm{~mm}$ en línea axilar media. Se procedió a realizar disección de ligamento esplenocólico con disector bipolar avanzado (Ligasure) para exponer polo inferior de bazo, posterior se continúa con ligamento gastroesplénico, ligamento frenocólico y, finalmente, lineofrénico. Se diseca ligamento pancreatoesplénico para exponer arteria y vena esplénica en el mismo hilio. Se realizó control vascular con endoengrapadora de $60 \mathrm{~mm}$ cartucho vascular (endoGIA), se introduce bolsa extractora por puerto de mano, se extrajo pieza quirúrgica íntegra por mismo puerto, se revisa adecuado control hemostático de lecho quirúrgico e hilio esplénico. Finalmente, se colocó drenaje cerrado hiperbárico tipo Jackson-Pratt (Biovac) hacia celdilla esplénica. Se cierra aponeurosis de puertos con ácido poliglicólico del número 1 y piel con polipropileno 000. Se reporta sangrado de $200 \mathrm{~mL}$ (sin la pieza quirúrgica) (Figura 3).

\section{Evolución}

La paciente evolucionó en posoperatorio inmediato de forma estable, con escala análoga del dolor 6 de 10 con analgesia convencional con paracetamol y ketorolaco, con gasto serohemático de drenaje cuantificado de $150 \mathrm{~cm}^{3}$ dentro de las primeras 24 a 48 horas, toleró la vía oral a las 12 horas de posoperatorio y mostró incremento en niveles de hemoglobina hasta $12 \mathrm{~g} / \mathrm{dL}$. Egresó a los cuatro días posoperatorios, para vigilancia en forma ambulatoria.

Reporte histopatológico macroscópico: Esplenomegalia de $25 \times 15 \times 10 \mathrm{~cm}$ de diámetro, con peso de 1,440 gramos. Microscópico: Congestión pasiva crónica (Figura 4).

Seguimiento a 12 meses con aumento en los niveles de hemoglobina y plaquetas, además de remisión del dolor abdominal. No complicaciones relacionadas con el abordaje o el procedimiento.

\section{DISCUSIÓN}

La cirugía de mínimo acceso fue referida por primera vez en el siglo X, cuando el médico Abulcasis utilizó la refracción de la luz para visualizar el cuello uterino; sin embargo, desde los siglos XIX y XX, cuando los procedimientos de invasión mínima se desarrollaron formalmente; ${ }^{4}$ múltiples centros a nivel internacional han desarrollado dispositivos y abordajes a fin de minimizar el riesgo de sangrado, disminución del dolor posoperatorio, de la estancia hospitalaria y los resultados estéticos, conservando estos beneficios de la cirugía de mínimo acceso. ${ }^{4,5}$ 
Las indicaciones actuales para la esplenectomía pertenecen primordialmente a patología hematológica y trauma; de esta primera, las indicaciones se dividen en benigna y maligna, correspondiendo hasta un $75 \%$ a la primera tabla de indicaciones de esplenectomía por patología hematológica. ${ }^{5}$

Así mismo, se han descrito indicaciones para la realización de la esplenectomía laparoscópica como: Patologías hematológicas benignas, patologías hematológicas malignas y quistes esplénicos. ${ }^{14}$ Dentro de las patologías hematológicas benignas, en las cuales la esplenectomía laparoscópica muestra ventajas significativas, encontramos la púrpura trombocitopénica idiopática (PTI), esferocitosis hereditaria, anemia hemolítica, entre otras. Se define como esplenomegalia masiva cuando el bazo rebasa la cresta iliaca y presenta un peso calculado de $1,500 \mathrm{~g}$, esto es resultado de múltiples patologías como hipertensión portal, leucemia o enfermedad de Gaucher, entre otras. ${ }^{14-16}$ Una opción para tratar quirúrgicamente esta entidad en casos de esplenomegalia masiva es la esplenectomía laparoscópica mano asistida, abordaje que busca la mínima pérdida sanguínea con una disección más precisa, incrementar los niveles de hemoglobina del paciente en el posoperatorio, conservando la cirugía de mínimo acceso, el mismo puerto de mano facilita la disección, además de ser el mismo sitio de extracción de pieza (Tabla 1). ${ }^{15-17}$

Se prefiere la esplenectomía laparoscópica para los tratamientos de enfermedades benignas y hematológicas secundarias a enfermedades de base, por mostrar ser un procedimiento seguro, baja morbilidad y ofrecer los beneficios de la cirugía de mínimo acceso, menor estancia hospitalaria, exposición quirúrgica, menor dolor y tiempo de recuperación. $3,5,14,15,17$
En los pacientes con esplenomegalia masiva, la cual se define en bazos con diámetros de $20 \mathrm{~cm}$ o más en su eje mayor, ${ }^{16,18}$ es un tema aún en controversia, debido a los múltiples retos relacionados con un bazo agrandado, como el espacio de trabajo limitado, dificultad en la retracción o movilización por las adherencias y riesgo de trauma vascular del hilio esplénico y la dificultad para la extracción de la pieza quirúrgica. En casos de esplenomegalia masiva se asocia a morbilidad posoperatoria que oscila entre $20-60 \%$, sin importar el tipo de abordaje, abierto o de mínimo acceso; esto asociado a las comorbilidades y enfermedades de base que llevaron a la esplenomegalia. ${ }^{16,17}$

Las principales causas de esplenomegalia son: Hipertensión portal por enfermedad hepática crónica, linfomas, leucemias y neoplasias mieloproliferativas, infecciones, trombosis de la vena esplénica y enfermedades hematológicas, éstas representan una indicación para esplenectomía laparoscópica si falla el tratamiento médico.

La Asociación Europea de Cirujanos Endoscopistas (EAES) en 2008 publicó las siguientes recomendaciones:2,19

1. Se prefiere el abordaje laparoscópico sobre el abierto para la mayoría de las indicaciones, ya que reduce las complicaciones y la recuperación es más corta.

2. La EL se recomienda para patologías benignas y malignas, y en caso de esplenomegalia, la cirugía puede acompañarse de mayores complicaciones, por lo que se requiere de mayor experiencia.

3. Durante la EL en patologías hematológicas autoinmunes, se recomienda realizar la búsqueda de tejido esplénico accesorio para evitar la recurrencia de la enfermedad.
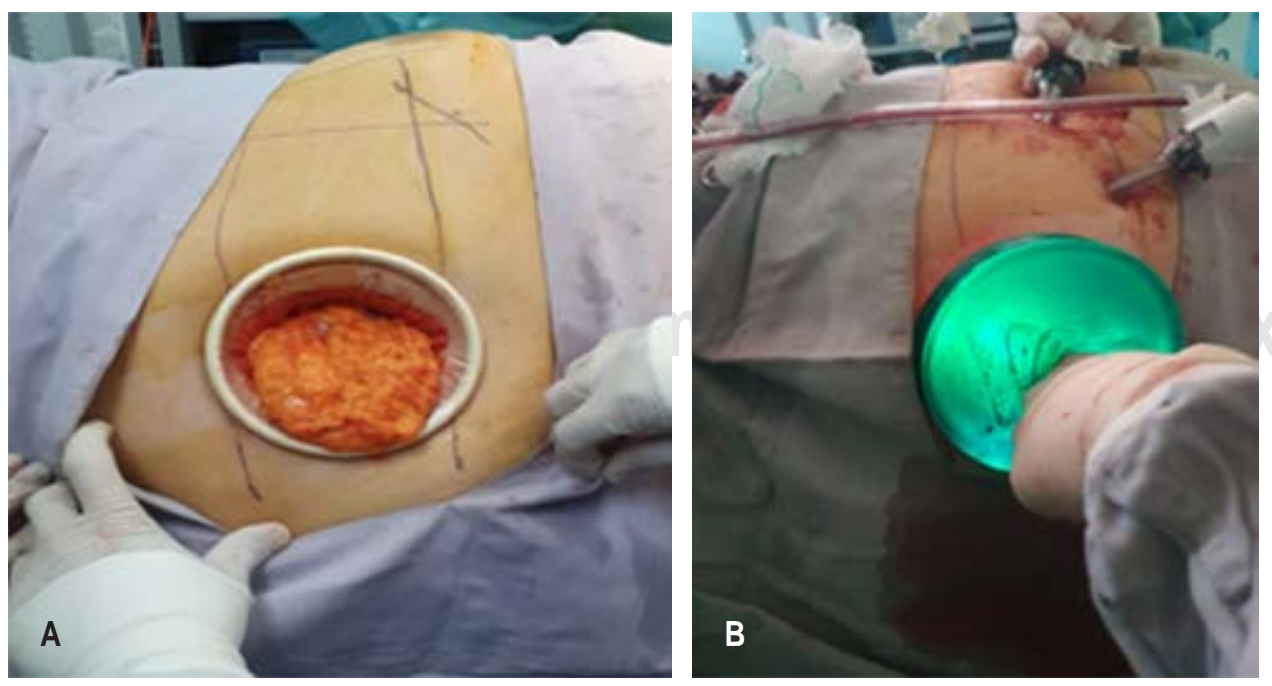

Figura 2:

A) Marcaje y colocación de Gel-port en flanco izquierdo para abordaje mano asistido de esplenectomía. B) Visualización in situ de puertos de trabajo para abordaje híbrido. 
Figura 3:

Retracción manual por puerto de laparoscopía mano asistida de la cara medial del bazo. A la izquierda, disección con energía ultrasónica en zona expuesta por retracción

manual.
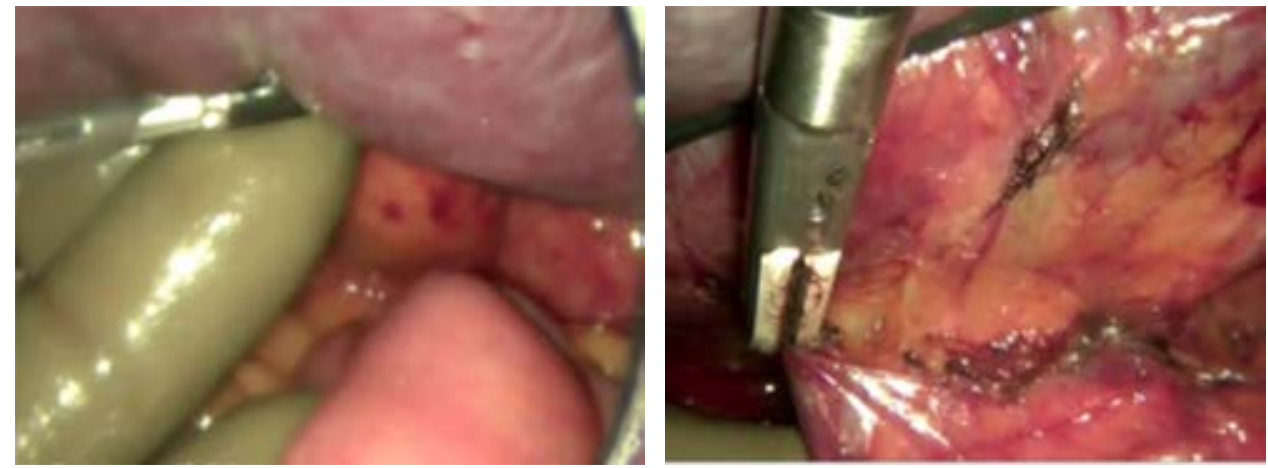

4. En pacientes con esplenomegalia (pero no esplenomegalia masiva), la EL es segura y preferible en manos experimentadas.

5. En la esplenomegalia masiva se debe considerar la esplenectomía laparoscópica asistida con mano o el abordaje abierto.

6. La hipertensión portal secundaria a cirrosis hepática es una contraindicación para la EL, al igual que la coagulopatía no corregida.

La esplenectomía laparoscópica mano asistida ha demostrado ventajas sobre la esplenectomía convencional en presencia de esplenectomía masiva, conserva la sensación táctil, la cual es útil para todo cirujano, facilita la movilización gentil del bazo, identificación hilio esplénico, la exposición de vasos cortos y del pedículo vascular para su disección, además de control en caso de eventual sangrado del mismo; sin perder las ventajas de la cirugía de mínimo acceso. ${ }^{2,18}$

El aumento relativo de tiempo quirúrgico, necesidad de más incisiones, sangrado, dolor posoperatorio y morbilidad demostraron que la esplenectomía mano asistida no mejora los resultados en tiempo quirúrgico, dolor posoperatorio y estancia hospitalaria comparada con la laparoscópica convencional y que la primera técnica tenía la desventaja de requerir incisiones adicionales. ${ }^{20}$

Litwin y colaboradores, en una serie multicéntrica, demostraron la utilidad de un puerto de mano en ocho casos de esplenomegalia. Estos pacientes no requirieron conversión a cirugía abierta y el promedio de estancia hospitalaria fue de 4.7 días, comparada con el reporte de Gossot, cuyo promedio fue de 3.7 días en pacientes sin esplenomegalia operados con la técnica laparoscópica tradicional; encontrando una estancia intrahospitalaria similar entre mano asistida y laparoscópica. 2,21

De igual forma, en la serie de Hellman y equipo ( $\mathrm{n}=$ 7) con esplenomegalia masiva (3.500-5.800 g) sometidos a esplenectomía laparoscópica mano asistida, el tiempo quirúrgico promedio fue de 133 minutos, las pérdidas sanguíneas de $300 \mathrm{~mL}$, un promedio de siete días de estancia hospitalaria y el dolor no fue reportado como severo, resultados muy alentadores, con conservación de los beneficios estéticos de la técnica laparoscópica convencional (Tabla 2). ${ }^{22}$

En tiempos recientes, existen documentos que describen el abordaje laparoscópico por incisión-única (SILS) con puertos de cuatro canales para la cirugía de bazo, entre otras. Sin embargo, su mayor uso se ha dado en pacientes en edad pediátrica con resultados similares a los de la laparoscopía convencional y/o mano asistida, además de no haber sido empleados en esplenomegalia masiva, por lo que no representa el abordaje de elección en casos de esta naturaleza. ${ }^{23}$

El sangrado transoperatorio supone uno de los mayores problemas y complicaciones durante una esplenectomía, con un promedio de $250 \mathrm{~mL}$. En la actualidad, existen varias opciones para la realización de hemostasia, entre las cuales se encuentran clips, suturas, coaguladores ultrasónicos o electroquirúrgicos (monopolares o bipolares). 2,6,24

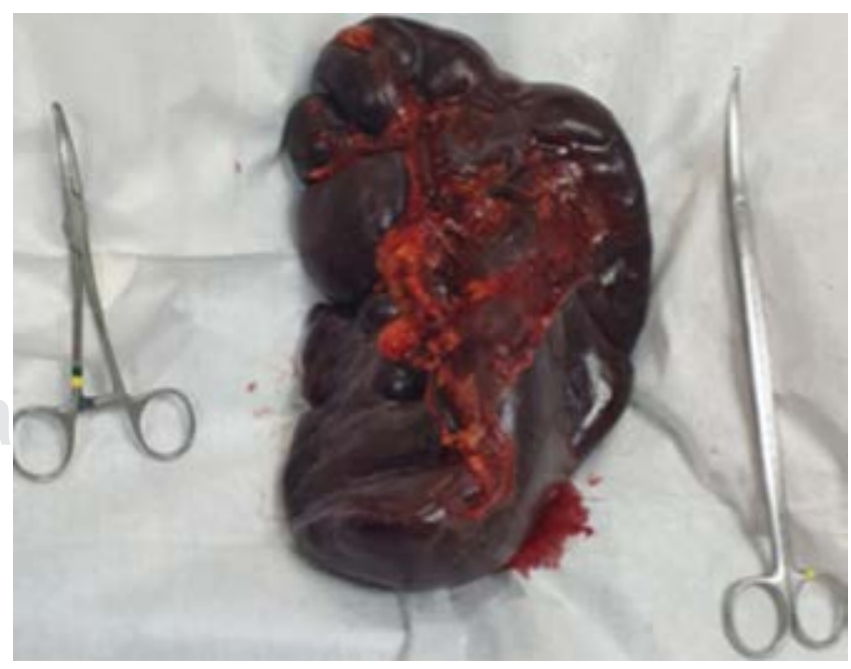

Figura 4: Pieza quirúrgica obtenida por Gel-port. Pinza de Kelly y tijera de Metzenbaun para referencia. 


\begin{tabular}{lll}
\hline \multicolumn{3}{c}{ Tabla 1: Indicaciones de esplenectomía } \\
en patología benigna.
\end{tabular}

Gelmini y colaboradores reportaron su serie $(n=$ 63) de pacientes, donde describieron que la utilización de Ligasure $^{\circledR}$ logra una reducción de tiempo quirúrgico por menor cambio de instrumentos, reducción de riesgos de complicaciones por electrocirugía, mínima adherencia de los tejidos al instrumento y escarificación, en los cuales sólo se requirió conversión por sangrado en tres casos. ${ }^{6}$

A pesar de los avances en los dispositivos y la selección de los pacientes, la morbilidad asociada a esplenectomía laparoscópica sigue siendo el sangrado, por lo cual se han propuesto nuevos métodos como embolización preoperatoria de la arteria esplénica en combinación con la esplenectomía laparoscópica asistida, reduciendo así tiempos quirúrgicos y sangrados, sobre todo en esplenomegalia masiva. ${ }^{7-9}$
La posición de elección de paciente para EL por diversos autores es el decúbito lateral derecho, con Trendelenburg inverso y flexión a $30^{\circ}$ para abrir el espacio entre la cresta iliaca izquierda y el margen costal. El puerto de mano se coloca en una incisión de 7-8 cm en línea media supraumbilical, y se coloca el resto de trocares (2-3) en la posición tradicional entre la cicatriz umbilical y el margen costal izquierdo. ${ }^{10}$ De igual forma, en la literatura se prefiere el abordaje lateral para la movilización esplénica, ya que permite una mejor exposición del hilio esplénico al desplazar el resto de las vísceras abdominales por gravedad. En nuestro caso, colocamos un puerto de visión de $10 \mathrm{~mm}$, y dos puertos de trabajo línea axilar anterior y media de 5 $\mathrm{mm}$, uno para el ayudante y la mano derecha del cirujano, pues la otra está en el puerto de mano.

En 2011, un estudio retrospectivo con 300 casos concluyó que el abordaje lateral se relacionaba con un tiempo quirúrgico menor (60 vs 80 minutos), un sangrado transoperatorio menor (30 vs $110 \mathrm{~mL}$ ) no tasas de conversión ( 0 vs $2.2 \%$ ), menos complicaciones posoperatorias (4.8 vs $31.5 \%)$ menor estancia intrahospitalaria (3.1 vs 5.2 días), menor dolor posoperatorio, y no formación de abscesos subfrénicos, comparados con el abordaje anterior. ${ }^{11}$

La elección actual es el uso de energía ultrasónica para la división del ligamento gastroesplénico y los vasos cortos. Al-Mulhim refiere variante con uso de Ligaclips para los vasos cortos de más de $4 \mathrm{~mm}$. Así mismo, parece que la variante más popular para manejo del hilio esplénico es el uso de una engrapadora con grapas vasculares una vez liberada la cola del páncreas para evitar lesión del mismo, con buenos resultados; algunas variantes incluyen el uso de energía ultrasónica, de instrumental quirúrgico tradicional con ligadura no absorbible. Existen, además, algunas variantes en la elección de la altura distal o proximal para el manejo de la arteria esplénica, su ligadura selectiva, entre otras. ${ }^{12,13}$

Por último, la obtención de la pieza presenta múltiples opciones, las cuales incluyen morcelado de la pieza dentro de una bolsa extractora, salida íntegra de la pieza

Tabla 2: Comparación de diversos estudios de esplenectomía por HALS.

\begin{tabular}{|c|c|c|c|c|}
\hline Estudio & Número de pacientes & $\begin{array}{l}\text { Tiempo operatorio } \\
\text { (minutos) }\end{array}$ & Conversión (\%) & $\begin{array}{l}\text { Estancia hospitalaria } \\
\text { (días) }\end{array}$ \\
\hline Meijer et al. & 22 & 89 & 4.5 & 3.9 \\
\hline Litwin et al. & 8 & 159 & 0.0 & 4.7 \\
\hline
\end{tabular}

HALS = hand assisted laparoscopic surgery. 
por incisión de puerto de mano o salida a través de una incisión tipo Pfannenstiel. En la literatura general, se sugiere el uso de drenajes en caso de sospechar lesión pancreática distal o ante el evento de sangrado abundante transoperatorio. ${ }^{10}$

Sclafani describió por primera vez su uso en la embolización arterial para esplenectomía en 1981. Existe la embolización arterial esplénica proximal (EAEP) y la embolización distal superselectiva. En la EAEP, el equivalente quirúrgico es la ligadura de la arteria esplénica, que fue reportada por primera vez en 1979. De igual manera, la EAEP promueve la hemostasia, causando una reducción en la presión sanguínea intraesplénica, lo que puede facilitar la formación de coágulos y disminución de aporte sanguíneo y de tamaño por hipoperfusión, lo que facilita el procedimiento y disminución de la morbilidad por sangrado. ${ }^{25,26}$

Poulin y colaboradores aplicaron la técnica de embolización prequirúrgica para reducir el tamaño esplénico y, de esta manera, mejorar la movilidad en especial de grandes bazos y disminuir hemorragia, la cual es el principal factor de conversión. ${ }^{27}$ De igual manera, la Guía de Práctica Clínica muestra como evidencia que la embolización bajo anestesia general, unas horas antes de la cirugía, ha tenido éxito, así como la embolización preoperatoria en la sala de operaciones bajo anestesia general inmediatamente antes de la cirugía, especialmente en caso de esplenomegalia masiva, para reducir tamaño y el sangrado transoperatorio..$^{25,28}$

Las complicaciones del curso posoperatorio varían del 0-35.7\%, según la serie. La mayoría de éstas comprenden sangrado, formación de abscesos subfrénicos, trombosis de la vena porta, isquemia intestinal, fuga pancreática, infección de herida quirúrgica y complicaciones respiratorias. Las tasas de conversión varían de $0-6.7 \% .{ }^{10}$ La incidencia de infección por agentes capsulados (Streptococcus pneumoniae, Neisseria meningitidis y Haemophilus influenzae tipo $B$ ) es cercana al $0.5 \%$ de los pacientes posesplenectomía anualmente; sin embargo, cursa con una tasa de mortalidad cercana al 50\%, por lo que la vacunación profiláctica en esencial en estos pacientes. ${ }^{12}$ Existe una entidad conocida como sepsis sideral o overwhelming post-splenectomy infection (OPSI), la cual consiste en una infección masiva por organismos capsulados, primordialmente $S$. pneumoniae con una incidencia de 0.13 casos por 1,000 pacientes, con una mortalidad entre $50-70 \%$, con un mayor riesgo relativo entre los dos y tres años posesplenectomía, la cual representa una verdadera urgencia médica que se manifiesta como vómito, diarrea, deterioro neurológico y sepsis y que requiere manejo oportuno. ${ }^{29,30}$

Se ha documentado una incidencia de $4.7-6.6 \%$ de trombosis de vena porta en pacientes posesplenectomía abierta. Se considera que la incidencia en técnicas laparoscópicas es similar. Hasta $87 \%$ de las mimas cursa asintomática; sin embargo, se considera una complicación potencialmente fatal. La esplenomegalia y un volumen esplénico total mayor de 1,300 g se consideran factores de riesgo independientes para su desarrollo. No se ha demostrado una relación directa con la trombocitosis secundaria y la incidencia de la misma. El uso de enoxaparina posoperatoria no ha demostrado disminución en la incidencia; no obstante, su empleo tras el diagnóstico demostró mejoría y resolución en casi todos los casos. La incidencia de la misma justifica el ultrasonido de seguimiento al día siete de la esplenectomía laparoscópica. ${ }^{10,12}$

\section{CONCLUSIÓN}

La valoración integral de las comorbilidades de los pacientes con esplenomegalias masivas asociadas a comorbilidades, facilita el tratamiento preoperatorio y optimización del paciente, y mejora los resultados por prevenir desarrollo de complicaciones.

La esplenectomía laparoscópica con puerto mano asistida es una opción indicada en pacientes con esplenomegalia masiva, porque permite adecuada movilización del bazo, estructuras adyacentes y provee un área de extracción de la pieza controlada por el mismo puerto; disminuye el índice de conversión quirúrgica a cirugía abierta, menor morbilidad y conserva los beneficios de la cirugía de mínimo acceso.

\section{REFERENCIAS}

1. Delaitre B, Maignien B. Splenectomy by the laparoscopic approach. Report of a case. Presse Med. 1991; 20: 2263.

2. Habermalz B, Sauerland S, Decker G, Delaitre B, Gigot JF, Leandros $\mathrm{E}$ et al. Laparoscopic splenectomy: the clinical practice guidelines of the European Association for Endoscopic Surgery (EAES). Surgl Endosc. 2008; 22: 821-848. Available in: https://doi.org/10.1007/s00464-007-9735-5.

3. Fisichella PM, Wong YM, Pappas SG, Abood GJ. Laparoscopic splenectomy: perioperative management, surgical technique, and results. Journal of Gastrointestinal Surgery. 2014; 18: 404-410. Available in: https://doi. org/10.1007/s111605-013-2366-3.

4. Filipi CJ, Fitzgibbons RJ, Salerno GM. In: Zucker KA, (ed). Historical review, diagnostic laparoscopy to laparoscopic cholecystectomy and beyond surgical laparoscopy. Quality Medical Publishing, Inc. St Louis Missouri. 1991, pp. 12-27.

5. Bonnet S, Guédon A, Ribeil JA, Suarez F, Tamburini J, Gaujoux S. Indications and outcome of splenectomy in hematologic disease. J Visc Surg. 2017; 154: 421-429. Available in: https://doi.org/10.1016/j. jviscsurg.2017.06.011.

6. Romano F, Caprotti R, Franciosi C et al. Laparoscopic splenectomy using ligasure-Preliminary experience. Surg Endosc. 2002; 16: 1608-1611.38. 
7. Lavu H. The feasibility and safety of laparoscopic splenectomy for massive splenomegaly: a comparative study. J Surg Res. 2012; 178: 119-120. Available in: https:// doi.org/10.1016/j.jss.2011.09.006.

8. Kim-Koha MJ, Souza-Gallardo LM. Esplenectomía laparoscópica como tratamiento para la púrpura trombocitopénica idiopática y la esplenomegalia masiva en un hospital de segundo nivel. Revista de la Facultad de Medicina de la UNAM. 2017. doi.org/10.22201. fm.24484865e.2018.61.6.03.

9. Weledji EP. Benefits and risks of splenectomy. Int J Surg. 2014; 12: 113-119. doi: 10.1016/j.ijsu.2013.11.017.

10. Moris D, Dimitriou N, Griniatsos J. Laparoscopic splenectomy for benign hematological disorders in adults: a systematic review. In vivo. 2017; 31: 291-302. Available in: https://doi.org/10.21873/invivo.11058.

11. Corcione F, Pirozzi F, Aragiusto G, Galante F, Sciuto A. Laparoscopic splenectomy: experience of a single center in a series of 300 cases. Surg Endosc. 2012; 26: 2870-2876. https://doi.org/10.1007/s00464-012-2272-x

12. Cadili A, De Gara C. Complications of splenectomy. Am J Med. 2008; 121: 371-375. Available in: https://doi. org/10.1016/j.amjmed.2008.02.014.

13. Keramidas D, Büyükünal C, Senyüz O, Dolatzas T. Splenic artery ligation: a ten-year experience in the treatment of selected cases of splenic injuries in children. Jpn J Surg. 1991; 21: 172-177.

14. Palandri F, Polverelli N, Sollazzo D, Romano M, Catani L, Cavo M, Vianelli N. Have splenectomy rate and main outcomes of ITP changed after the introduction of new treatments? A monocentric study in the outpatient setting during 35 years. Am J Hematol. 2016; 91: E267-E272. Available in: https://doi.org/10.1002/ajh.24310.

15. Somasundaram SK, Massey L, Gooch D, Reed J, Menzies D. Laparoscopic splenectomy is emerging "gold standard" treatment even for massive spleens. Ann R Coll Surg Engl. 2015; 97: 345-348. Available in: https://doi.org/10.1308/0 03588414 X14055925060479.

16. O'Reilly RA. Splenomegaly in 2,505 patients in a large university medical center from 1913 to 1995.1913 to 1962: 2,056 patients. West J Med. 1998; 169: 78-87.

17. Bellows CF, Sweeney JF. Laparoscopic splenectomy: present status and future perspective. Expert Rev Med Devices. 2006; 3: 95-104. Available in: https://doi. org/10.1586/17434440.3.1.95.

18. Di Franco G, Gianardi D, Bianchini M, Palmeri M, Morelli L. The role of hand-assisted laparoscopic splenectomy for mega spleens in the da Vinci era. J Robot Surg. 2019; 13:
791-792. Available in: https://doi.org/10.1007/s11701-01900985-4.

19. Casaccia M, Sormani MP, Palombo D, Dellepiane C, Ibatici A. Laparoscopic splenectomy versus open splenectomy in massive and giant spleens: should we update the 2008 EAES Guidelines? Surg Laparosc Endosc Percutan Tech. 2019; 29: 178-181. Available in: https://doi.org/10.1097/ SLE.0000000000000637.

20. Meijer DW, Gossot D, Jakimowicz JJ, De Wit LT, Bannenberg JJ, Gouma DJ. Splenectomy revised: manually assisted splenectomy with the dexterity device--a feasibility study in 22 patients. J Laparoendosc Adv Surg Tech A. 1999; 9: 507510. doi: 10.1089/lap.1999.9.507.

21. Litwin DE, Darzi A, Jakimowicz J, Kelly JJ, Arvidsson D, Hansen P et al. Hand-assisted laparoscopic surgery (HALS) with the HandPort system: initial experience with 68 patients. Ann Surg. 2000; 231: 715-723. doi: 10.1097/00000658-200005000-00012.

22. Hellman P, Arvidsson D, Rastad J. HandPort-assisted laparoscopic splenectomy in massive splenomegaly. Surg Endosc. 2000; 14 (12): 1177-1179. doi: 10.1007/ s004640000210.

23. Gkegkes ID, Mourtarakos S, Iavazzo C. Single-incision laparoscopic splenectomy. JSLS. 2014; 18: e2014.00350. doi: 10.4293/JSLS.2014.00350.

24. Gelmini R, Romano F, Quaranta N, Caprotti R, Tazzioli G, Colombo $G$ et al. Sutureless and stapleless laparoscopic splenectomy using radiofrequency: LigaSure device. Surg Endosc. 2006; 20: 991-994. Available in: https://doi. org/10.1007/s00464-005-0470-5.

25. Bessoud B, Denys A. Main splenic artery embolization using coils in blunt splenic injuries: effects on the intrasplenic blood pressure. Eur Radiol. 2004; 14: 1718-1719.

26. Sclafani SJ. The role of angiographic hemostasis in salvage of the injured spleen. Radiology. 1981; 141: 645-650.

27. Poulin E, Thibault C, Mamazza J, Girotti M, Coté G, Renaud A. Laparoscopic splenectomy: clinical experience and the role of preoperative splenic artery embolization. Surg Laparosc Endosc. 1993; 3: 445-450.

28. Esplenectomía. Guía de Evidencias y Recomendaciones: Guía de Práctica Clínica México. CENETEC; 2015.

29. Tahir F, Ahmed J, Malik F. Post-splenectomy sepsis: a review of the literature. Cureus. 2020; 12: e6898. doi: 10.7759/ cureus.6898.

30. Luu S, Spelman D, Woolley IJ. Post-splenectomy sepsis: preventative strategies, challenges, and solutions. Infect Drug Resist. 2019; 12: 2839-2851. Available in: https://doi. org/10.2147/IDR.S179902. 